\title{
Correspondence
}

\section{Comment Regarding Increased Striatal Glutamate in Schizophrenia}

\author{
Richard J Maddock*, 1,2 and Michael H Buonocore ${ }^{1,3}$ \\ IImaging Research Center, University of California Davis Medical Center, Sacramento, CA, USA; '2Department of Psychiatry, University \\ of California Davis Medical Center, Sacramento, CA, USA; ${ }^{3}$ Department of Radiology, University of California Davis Medical Center, \\ Sacramento, CA, USA
}

Neuropsychopharmacology (2012) 37, 1067-1068; doi:10.1038/npp.201 I.180

de la Fuente-Sandoval et al, (2011) recently reported that MRS-measured striatal glutamate is significantly higher in first-episode schizophrenia patients and ultra high-risk subjects compared with controls. They also report significant positive correlations between glutamate and NAA levels in striatum and cerebellum.

This theoretically motivated study of antipsychotic naïve patients is applauded for its potential to further our understanding of schizophrenia, but it is important to point out a critical limitation of the quantitative methods that appears to invalidate the conclusions. The authors have used the 'absolute concentration' values for metabolites generated by LCModel, which are listed in the first column of its output. Section 2.1 of the LCModel manual, version 6.2-3 (Provencher, 1993) specifically cautions against using these values in statistical analyses without first applying a unique correction factor to all metabolites from each MRS acquisition. The manual states that only if techniques such as waterscaling or creatine normalization are used, will the arbitrarily scaled absolute concentration data be meaningful. It appears that the authors used these values without applying one of these techniques.

Patient and MRI system factors, such as head size and position within the eight-channel coil affecting induced voltage in the coil elements, and drift and variability of intrinsic gain in the amplifiers of the RF receiver system, substantially confound the uncorrected 'absolute concentration' values. The group differences observed in de la Fuente-Sandoval et al may be due mainly to these patient and MRI system factors, not to differences in metabolite concentrations.

Only group differences revealed after the appropriate scaling or normalization of the data may be attributed to metabolite concentration differences. Fortunately, the

*Correspondence: Professor RJ Maddock, Imaging Research Center, Department of Psychiatry, University of California Davis, $4701 \times$ Street, Sacramento, CA 95817, USA, Tel: + I 916734 3286, Fax: + I 916 734 8750, E-mail: rjmaddock@ucdavis.edu

Received 26 July 201 I; accepted 28 July 201 I authors have already acquired the data necessary to apply either creatine normalization or water-scaling as described in the LCModel manual. It is important to note that in all of the 15 MRS glutamate studies summarized by de la FuenteSandoval et al, (2011) in their Table 3, one or the other of these two techniques was applied.

de la Fuente-Sandoval et al, (2011) also report significant correlations between glutamate and NAA in each examined group and brain region. Unfortunately, this correlation analysis is confounded by the patient and system factors referred to above. These factors effectively cause the entire spectrum from each MRS acquisition to be multiplied by a unique, but unknown, number. Consequently, within each spectrum, all 'absolute concentration values', including those of glutamate and NAA, are inherently correlated, that is, they increase and decrease in unison, depending upon the unique number multiplying each spectrum. Thus, when evaluating for a biologically meaningful correlation between the glutamate and NAA values, the null hypothesis must be a positive number reflecting the inherent correlation, not zero. As de la Fuente-Sandoval et al, (2011) used a null hypothesis of zero correlation, their statistical analysis cannot support conclusions about biologically meaningful correlations. Creatine normalization or waterscaling can reduce the inherent correlation among metabolite 'concentration values,' but not completely eliminate it. Even with these techniques, investigators must be cautious about the interpretation of correlations between metabolites measured during the same MRS acquisition.

\section{DISCLOSURE}

The authors declare that over the past 3 years, RJM has received compensation from Pfizer, a manufacturer of antipsychotic medications. Other than University funds and $\mathrm{NIH}$ grant support, the authors have received no other support or compensation from individual or corporate entities that could be perceived as constituting a conflict of interest. 


\section{REFERENCES}

de la Fuente-Sandoval C, Leon-Ortiz P, Favila R, Stephano S, Mamo D, Ramirez-Bermudez J et al. (2011). Higher levels of glutamate in the associative-striatum of subjects with prodromal symptoms of schizophrenia and patients with first-episode psychosis. Neuropsychopharmacology 36: 1781-1791.

Provencher SW (1993). Estimation of metabolite concentrations from localized in vivo proton NMR spectra. Magn Reson Med 30: 672-679. 